\title{
Mathematical modelling of aliphatic glucosinolate chain length distribution in Arabidopsis thaliana leaves
}

\author{
Beate Knoke $\cdot$ Susanne Textor $\cdot$ \\ Jonathan Gershenzon · Stefan Schuster
}

Received: 6 February 2008/Accepted: 5 July 2008/Published online: 16 August 2008

(C) The Author(s) 2008. This article is published with open access at Springerlink.com

\begin{abstract}
Aliphatic glucosinolates are a major class of defensive secondary metabolites in plants that are mostly derived from methionine. Occurring in different chain lengths, they show a structural diversity arising from the variable number of chain elongation cycles taking place during their biosynthesis. The key enzymes in determining glucosinolate chain length are the methylthioalkylmalate (MAM) synthases, MAM1 and MAM3, with MAM3 showing a broader substrate specificity than MAM1. A comparison of the measurements of wild type and MAM1 knockout mutant plants shows the following distinct changes in glucosinolate chain length profiles:

(1) a reversal of the relative proportions of the two shortest glucosinolates,

(2) a significant increase in the concentration of the longest glucosinolate,
\end{abstract}

B. Knoke $\cdot$ S. Schuster

Department of Bioinformatics, Friedrich Schiller

University, Ernst-Abbe-Platz 2, 07743 Jena, Germany

Present Address:

B. Knoke $(\square)$

Department of Biochemical Engineering, University of Stuttgart, Allmandring 31, 70569 Stuttgart, Germany

e-mail: knoke@ibvt.uni-stuttgart.de

\section{S. Textor $\cdot$ J. Gershenzon}

Department of Biochemistry, Max Planck Institute for Chemical Ecology, Hans-Knöll-Strasse 8, 07745 Jena, Germany
(3) an increase in total glucosinolate content in the mutant.

MAM3 knockout mutants on the contrary differ from wild type plants by a pronounced abundance of the second shortest glucosinolate and the depletion of the two longest glucosinolates. To clarify the contribution of the multifunctional enzymes MAM1 and MAM3 to the glucosinolate profile of Arabidopsis thaliana leaves, we simulated glucosinolate biosynthesis in a kinetic model, taking into account the structure of the pathway and measured enzymatic properties. The predicted glucosinolate profiles show all characteristics of the actual differences between wild-type and MAMI mutants or MAM3 mutants, respectively. The model strongly supports experimental indications that the two MAM activities are not independent of each other. In particular, it showed that an elevated expression of MAM3 in the MAM1 mutant is critical in determining the glucosinolate profile of this plant line. The simulation was critical for this finding since it allowed us to assess the individual effects of two processes-the knocking out of MAM1 and the overexpression of MAM3 - that are difficult to separate experimentally.

Keywords Kinetic modelling - Plant defense metabolites - Simulation of metabolism . Methylthioalkylmalate synthase $\cdot$ Methionine chain elongation 


$\begin{array}{ll}\text { Abbreviations } & \\ \text { MAM1 } & \text { Methylthioalkylmalate synthase 1 } \\ \text { MAM3 } & \text { Methylthioalkylmalate synthase 3 } \\ \text { MTOB } & \text { 4-Methylthio-2-oxobutanoate } \\ \text { MTOP } & \text { 5-Methylthio-2-oxopentanoate } \\ \text { MTOH } & \text { 6-Methylthio-2-oxohexanoate } \\ \text { MTOHp } & \text { 7-Methylthio-2-oxoheptanoate } \\ \text { MTOO } & \text { 8-Methylthio-2-oxooctanoate } \\ \text { MTON } & \text { 9-Methylthio-2-oxononanoate } \\ \text { MTOD } & \text { 10-Methylthio-2-oxodecanoate } \\ \mathrm{C}_{3} & \text { 3-Methylthiopropylglucosinolate } \\ \mathrm{C}_{4} & \text { 4-Methylthiobutylglucosinolate } \\ \mathrm{C}_{5} & \text { 5-Methylthiopentylglucosinolate } \\ \mathrm{C}_{6} & \text { 6-Methylthiohexylglucosinolate } \\ \mathrm{C}_{7} & \text { 7-Methylthioheptylglucosinolate } \\ \mathrm{C}_{8} & \text { 8-Methylthiooctylglucosinolate } \\ \mathrm{CYP79F}_{2} & \text { Two cytochrome P450 } \\ \text { and CYP79F2 } & \text { Homomethionine monooxygenases } \\ & \end{array}$

\section{Introduction}

In modern quantitative biochemistry, the combination of experimental and computational approaches is becoming more and more important (cf. Heinrich and Schuster 1996; Fell 1997; Fong and Palsson 2004). It is a trademark of modern systems biology (cf. Klipp et al. 2005) that experiment and modeling are combined in a synergistic way. Due to the complexity of most metabolic networks, understanding their behavior intuitively is next to impossible, all the more when multifunctional enzymes are involved. Approaches have been developed using kinetic models involving differential equations, with each differential equation describing the rate of change in one metabolite concentration. The temporal changes of metabolite concentrations are calculated by numerical integration of their differential equations. At steady state, the metabolite concentrations do not change anymore, and so the differential equations can be set equal to zero and, therefore, simplified to give algebraic equations. In a reaction network at steady state, the participating enzymes exert a certain amount of control over the magnitudes of the metabolic fluxes and the levels of the metabolites in the system. The quantification of this control for each enzyme in the network in steady state can be expressed in control coefficients. Those coefficients were defined within the theory of Metabolic Control Analysis, which was developed by Kacser and Burns (1973) and Heinrich and Rapoport (1973, 1974) (cf. Heinrich and Schuster 1996). Control coefficients are systemic properties, which are influenced by network structure, the kinetic parameters of enzymes and the equilibrium constants of reactions.

Plant metabolism has repeatedly been the subject of kinetic modeling, for reviews see Morgan and Rhodes (2002) and Lange (2006). Here, we present a kinetic model of the synthesis of aliphatic glucosinolates in Arabidopsis thaliana leaves (in particular, its Columbia ecotype). Glucosinolates, the subject of this special issue, are one of the largest groups of secondary metabolites in A. thaliana (Halkier and Gershenzon 2006; Grubb and Abel 2006) and serve as antiherbivore defenses (Wittstock et al. 2003). These amino acid-derived substances have variable side chains and are linked to a glucose residue (Fahey et al. 2001) (Fig. 1). Besides variation in chain length, different precursor amino acids and diverse patterns of secondary oxidation and esterification contribute to the diversity of glucosinolates (Tokuhisa et al. 2004; Kliebenstein, this issue). The largest group of glucosinolates in A. thaliana is biosynthesized from methionine, which is initially transaminated to a 2-oxo-acid. Subsequently, the 2-oxo-acid is subject to repeated chain elongation, followed by the formation of the glucosinolate core and side chain modifications (Wittstock and Halkier 2002). Chain elongation is the first phase of glucosinolate formation and is thus responsible for redirecting the amino acid flux from primary to secondary metabolism.

Glucosinolates of six different chain lengths, referred to as a $\mathrm{C}_{3}$ to $\mathrm{C}_{8}$ glucosinolates, are produced in A. thaliana, due to a variable number of iterative chain elongation cycles, each adding one methylene carbon to the molecule (Graser et al. 2000). In the Columbia ecotype, the first reaction of the three-step chain elongation cycle, the condensation of a 2-oxo acid with acetyl-CoA, is catalyzed by one of two methylthioalkylmalate synthase enzymes: MAM1 and MAM-L. MAM1 acts principally on the 2-oxoacids with the two shortest chain lengths and with a very weak activity on the third one (Textor et al. 2004; Benderoth et al. 2006), while MAM-L acts on all six 2-oxo-acids present (Textor et al. 2007). Textor et al. (2007) proposed renaming MAM-L as $M A M 3$, based on the biochemical properties of the 
Fig. 1 The pathway of methionine-derived glucosinolate biosynthesis in A. thaliana can be divided into the methionine chain elongation cycle and the biosynthesis of the core glucosinolate structure. The parent methylthioalkyl glucosinolates can undergo further side chain modifications. For clarity, intermediates are shown in the form of substance classes of different chain lengths. For A. thaliana, $n$ ranges from 1 to 6

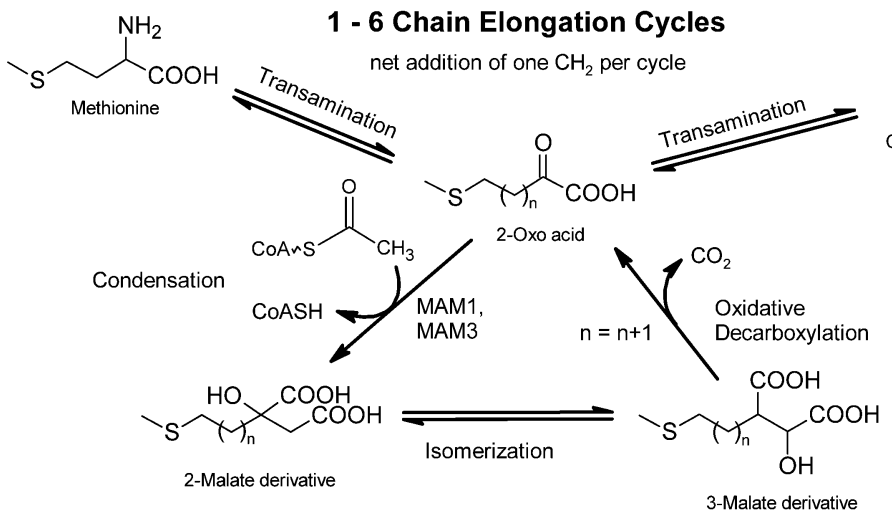

Formation of Glucosinolate Core

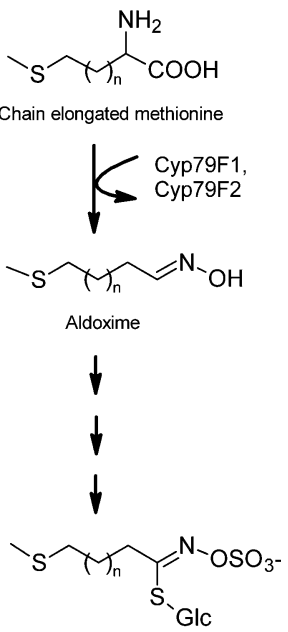

Parent glucosinolate encoded enzyme and the earlier naming of MAM1 and MAM2 (Kroymann et al. 2003). For a detailed discussion on the evolution of the genes encoding the MAM enzymes, please see Benderoth et al., this issue. The MAM synthases belong to a large enzyme family condensing various 2-oxo acids with an acylCoA ester (Textor et al. 2004). The broad substrate specificity of MAM3 and the fact that the kinetic parameters for substrates of varying chain length form a non-monotonic series (see Table III in Textor et al. 2007) raised the question of how these properties contribute to the complex glucosinolate distribution and their changes observed in vivo. Unfortunately, the nonlinearity of Michaelis-Menten kinetics and the convoluted structure of the biosynthetic network make the system so complex that intuitive reasoning is insufficient to explain the observed profiles.

The chain-length spectrum of aliphatic glucosinolates in $A$. thaliana is critically influenced by metabolic partitioning at the branchpoint between diversion into glucosinolate core biosynthesis or further elongation. The first step after the branchpoint in further elongation is an irreversible reaction catalyzed by the methylthioalkylmalate (MAM) synthases. These likely exert flux control. The first step in the diversion is a transamination which is fast and reversible. As fast, reversible reactions exert virtually no control on metabolic flux (cf. Heinrich and Schuster 1996; Kholodenko et al. 1998), the relevant step for understanding control at this branchpoint is the second reaction, the conversion of the elongated amino acid to an oxime, catalyzed by CYP79F1 (for all chain lengths) and CYP79F2 (for the two longest chains). The latter two enzymes are again likely to exert flux control.

Despite knowledge of the substrate kinetics of the chain elongation cycle enzymes MAM1 and MAM3, mutations in the corresponding genes lead to glucosinolate profiles differing from those of wild-type plants that are not fully explainable: MAM3 missense or knockout mutants show almost no change in $\mathrm{C}_{3}$ glucosinolates and only a slight increase in $\mathrm{C}_{4}$, but have a complete lack or much reduced level of longchain glucosinolates $\mathrm{C}_{6}-\mathrm{C}_{8}$, which can be restored by transgenic expression of MAM3 (Field et al. 2004; Textor et al. 2007). MAM1 mutants also show an interesting glucosinolate profile: whereas $\mathrm{C}_{4}$ glucosinolates are dominant in wild-type plants, in MAM1 missense or knockout mutants $\mathrm{C}_{3}$ glucosinolates are more abundant (Haughn et al. 1991; Kroymann et al. 2001; Textor et al. 2007). The glucosinolate profile of a plant line in which the MAMI gene is disrupted by a T-DNA insertion (gsml-3) was examined by Textor et al. (2007). In addition to a large reduction in $\mathrm{C}_{4}$ and a concomitant increase in $\mathrm{C}_{3}$ glucosinolates, it shows two other differences in comparison to the wild-type (Fig. 3a): a moderate increase in $\mathrm{C}_{8}$ glucosinolates and an increase in total aliphatic glucosinolates. To elucidate the mechanisms leading 
to these changes in the glucosinolate profile was another motivation for constructing a mathematical model.

The model uses pathway architecture and experimentally determined enzyme kinetic data to evaluate the contribution of MAM1 and MAM3 to the glucosinolate profile and to determine how the properties of both enzymes affect the diversity of aliphatic glucosinolate chain lengths in A. thaliana leaves. To test the reliability of our model, we conducted a simulation not only for the Columbia wild type, but also for the MAM1 mutant gsm1-3 and the MAM3 missense mutant gsm2-1 (Textor et al. 2007). When kinetic data were not known, we chose values to make the agreement of the model predictions with the actual distribution of different chainlength glucosinolates as good as possible. Due to the uncertainty of these parameters, the model is not an exact quantitative simulation, but attempts to explain the observed glucosinolate distribution in a more qualitative manner.

\section{Model description}

\section{The reaction network}

Figure 1 depicts a cyclic architecture for the glucosinolate chain elongation pathway. However, while elongation is cyclic from the point of view of the enzymes, it is not from the viewpoint of the metabolites since the product of the "cycle" is not identical to the starting substrate, but involves an additional methylene group. Thus in this model we depict the pathway as a branched acyclic network (Fig. 2) to accurately assess the concentration profile of the different metabolites.

To reduce the complexity of the model, most of the enzymes of the pathway are not considered separately. Such "skeleton models" consisting of few overall reactions have turned out to be very useful in previous studies (cf. Selkov 1975; Heinrich and Schuster 1996). The first transamination of methionine leading to its 2oxo-acid MTOB (see list of abbreviations) was left out as transamination reactions are usually fast and reversible and so can be regarded to be in rapid equilibrium. Therefore, the concentration of MTOB is assumed to be in equilibrium with methionine, and MTOB was considered as the initial substrate with a constant concentration (designated as an external metabolite for purposes of the model). The same reasoning applies to the transamination reactions converting the chain-elongated 2-oxo-acids to the elongated homomethionine derivatives which are also assumed to be in quasi-equilibrium.

Some more simplifications can be achieved by applying the principles of Metabolic Control Analysis (cf. Heinrich and Schuster 1996), applicable whenever the system is at steady state. Important in this respect are particularly the structure of the network, e.g. the branching pattern, and the reversibility or irreversibility of the catalyzed reactions. If we assume a buffered concentration of the substrate pool for a reaction network branch, the enzyme catalyzing the first irreversible reaction exerts full control over the magnitude of the metabolic flux in that branch if there is no feedback from downstream intermediates or signaling to that enzyme (Heinrich and Rapoport 1974, cf. Heinrich and Schuster 1996). Then all subsequent enzymes can be considered to exert virtually no control over flux, so that the branch is effectively "shortened".

In glucosinolate chain elongation, the condensation reactions catalyzed by MAM1 and MAM3 are irreversible because they involve hydrolysis of the high energy thioester bond of an acetyl-CoA (Textor et al. 2004, 2007). Following the "skeleton model" approach, we have neglected the other two steps of the chain elongation cycle, isomerization and oxidative decarboxylation, in our kinetic modeling. These are incorporated into the condensation reactions, thus reducing complexity and parameter number. This simplification is justified by the fact that the MAM1 and MAM3 reactions are the first irreversible reactions in the pathway, so that they fully control the overall metabolic flux through glucosinolate biosynthesis, if we assume a constant concentration of methionine. A further reason for the lack of control at the isomerization step is that isomerization reactions are nearly always fast because they are monomolecular. The three reaction chain-elongation sequence was therefore represented by the condensation reaction catalyzed by the MAM enzymes, which was denoted by $\mathrm{E}_{i}$ (Fig. 2), with $i$ standing for the index of the elongation step. Whereas the enzyme catalysing the first irreversible reaction determines the magnitude of the overall flux through a branched pathway, downstream enzymes can control the distribution of this flux through diverging sub-branches. 
Fig. 2 Simplified representation of the pathway of aliphatic glucosinolate biosynthesis used in model construction. The branched acyclic network arises from considering the chain elongation process as a series of sequential events. Simplification was achieved by reducing each chain elongation cycle into one step, $\mathrm{E}_{i}$, and reducing the conversion of chain elongated 2-oxo acids (MTOP, MTOH...) into the final glucosinolates $\left(\mathrm{C}_{3}\right.$, $\mathrm{C}_{4} \ldots$ ) into one step, $\mathrm{G}_{i}$, as described in the text. Furthermore, the initial deamination starting from methionine is incorporated into the first condensation step. To reach a steady state level of glucosinolates, a hypothetical degradation step was included. For abbreviations, see text
Chain Elongation<smiles>CSCCC(=O)C(=O)O</smiles><smiles>FC(F)(F)F</smiles><smiles>CSCCCC(=O)C(=O)O</smiles><smiles>FC(F)(F)F</smiles><smiles>CSCCCCC(=O)C(=O)O</smiles><smiles>FC(F)(F)F</smiles><smiles>CSCCCCCC(=O)O</smiles>
$E_{4}$<smiles>CSCCCCCCC(=O)C(=O)O</smiles>
$E_{5}$<smiles>CSCCCCCCCC(=O)C(=O)O</smiles><smiles>CSCCCCCCCCC(=O)C(=O)O</smiles>

Transamination and Glucosinolate Core Formation

Degradation
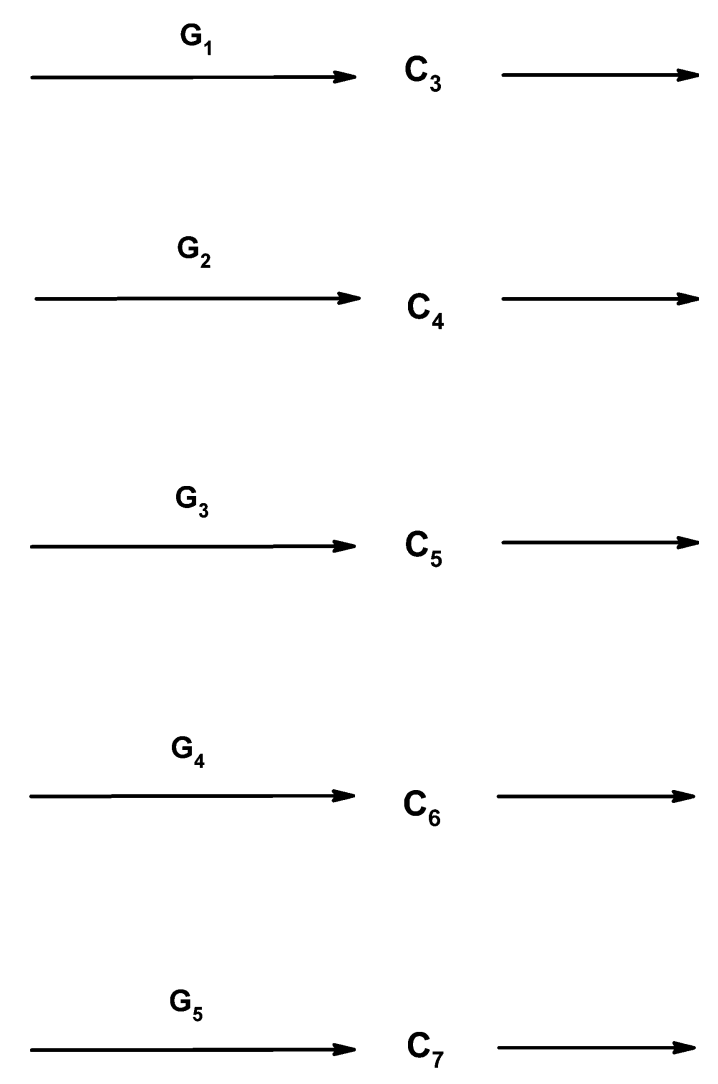

Similar reasoning was applied for the sub branch of glucosinolate core formation: The CYP79F1 and F2 monooxygenases catalyze the formation of an aldoxime from homomethionine in an oxidative decarboxylation, which can be considered nearly irreversible because of the formation of (gaseous) $\mathrm{CO}_{2}$ and the consumption of oxygen. Therefore, we neglect the enzymes downstream of CYP79F1 and F2, as they likely exert almost no control on the flux distribution into their sub-branch. The six different reactions of core glucosinolate formation catalysed by CYP79F1 (two of which are also catalysed by CYP79F2) are here denoted as $\mathrm{G}_{1}-\mathrm{G}_{6}$ : They include, moreover, the reamination reactions, which are in quasi-equilibrium.

An interesting point is how control is distributed at the branch points in the network (see Fig. 2). Control over the total flux is almost entirely exerted by the MAM enzymes upstream of the first branch point. By contrast, as alluded to above, both the MAM enzymes 
and the CYP79F1 and F2 enzymes exert control over the flux ratios between further elongation and diversion into glucosinolate core biosynthesis.

In addition to the formation of the different chainlength glucosinolates, degradation reactions were included in the model in order to endow the glucosinolate products with a stationary state. Without the inclusion of such degradation reactions, which may also be considered to represent dilution by cell growth, the glucosinolates would permanently accumulate in the model making the calculations more cumbersome.

Rate laws and kinetic parameters

The condensation reactions $\mathrm{E}_{i}$, leading to chainelongated 2-oxo acids, and the reactions from the chain-elongated homomethionines to the final glucosinolates were modeled by irreversible MichaelisMenten rate laws (which have been measured for MAM3 and assumed for MAM1, CYP79F1 and F2),

$v=\frac{V_{\max } * S}{K_{m}+S}$.

As the transamination reactions between these two steps were considered to be in quasi-equilibrium, their equilibrium constants were combined with the following glucosinolate-formation steps in one reaction. Quasi-equilibrium implies that we can use the massaction law:

$P=q * T$

with $q$ being the equilibrium constant and $P$ and $T$ denoting the concentrations of extended analogs of methionine and MTOP or its extended analogs, respectively. This can be inserted into Eq. 1 with $S$ replaced by $P$ :

$v=\frac{V_{\max } * q * T}{K_{m}+q * T}=\frac{V_{\max } * T}{\frac{K_{m}}{q}+T}$

Reaction velocities and concentrations of $T$ are calculated automatically during time course and steady state calculation.

A serious problem is that the maximal velocities, $V_{\text {max }}$, are unknown because they are proportional to the enzyme concentrations. We circumvented this problem by making use of the fact that most of the enzymes involved in this network are multifunctional so that the enzyme concentration is the same for each particular reaction catalysed by the same enzyme. Moreover, it should be noted that scaling all reaction rates in a metabolic network by the same factor leaves all steadystate metabolite concentrations unchanged (cf. Heinrich and Schuster 1996; Fell 1997). Instead of $V_{\max }$ values for MAM3, we started from the values that are usually measured in experiment-the turnover numbers normalized by the molar mass of the enzyme, that is, the maximal velocity normalized by mass concentration of the enzyme (Table III in Textor et al. 2007). As the mass concentration of MAM3 is unknown, we rescaled all experimentally measured, normalized maximal velocities, such that the virtual mass concentration of MAM3 in the wild-type is $1 \mu \mathrm{g}^{-1}$. This choice is equivalent to a scaling by the unknown mass concentration of MAM3. Practically, this meant that we multiplied the measured values in the enzyme assay of nmol $\mathrm{min}^{-1} \mathrm{mg}^{-1}$ by a MAM3 concentration of $1 \mu \mathrm{g}^{-1}$ and changed the unit into nmol $\min ^{-1} 1^{-1}$. This rescaled normalized $V_{\max }$ is here denoted by $V_{\max }^{\prime}$. Explaining this rescaling in another way, it can be said that from the measured activities of MAM3, we can see the relative activities of that enzyme on the various chains lengths rather than the absolute $V_{\max }$ values. Nevertheless, this is sufficient for calculating the relative glucosinolate chain-length distributions. Since, in the model, we need to take some absolute values of kinetic parameter values, we did the rescaling explained above, keeping in mind that only relative values are known. Another variant would be to keep the turnover numbers normalized by the molar mass as they have been measured and scale the maximal velocities and rate constants of all the other reactions accordingly. However, we believe it is easier to think in terms of maximal velocities, even if they are based on assumed enzyme concentrations.

The maximal velocities of MAM1, CYP79F1 and CYP79F2 and the rate constants of the degradation of glucosinolates are fitted rather than measured, so that we can decide to fit them as scaled values. However, in the MAM1 knockout mutant, to be discussed below, the concentration of MAM3 differs from that in the wild-type by a factor $x$ to be determined later. Thus, the observed difference in the transcript levels is translated into a difference in the enzyme concentration and, thus, in $V_{\max }^{\prime}$. In this case, $V_{\max }^{\prime}$ of MAM3 from the wild type is scaled to correspond to a mass concentration of $x \mu \mathrm{g}^{-1}$. In light of the way the model is constructed, we cannot directly compare the absolute contents of glucosinolates per gram dry 
weight with those present in the plant. However, we can compare the relative distributions, and likewise the change of distribution between wild-type and mutant lines.

Table 1 shows the kinetic parameters and the initial (fixed) concentration of MTOB used in the rate laws. Kinetic values set in Roman type were obtained from Textor et al. $(2004,2007)$, or, in the case of the CYP79F1/F2 enzymes, from Chen et al. (2003). Values set in italics were derived from assumptions given above or chosen from preliminary simulations to optimize correspondence of the model with actual glucosinolate chain-length distributions observed.

Kinetic values for some reactions were not considered in the model. Branched-chain Aminotransferase 4, which has been shown to catalyze the transamination of methionine to MTOB follows a reversible Michaelis-Menten kinetics (Schuster et al. 2006a, b), and both $V_{\max }$ and $K_{m}$ values were reported. However, since there are six further transamination reactions, an inclusion of these measured values in the model would mean having to represent the following six

Table 1 Overview of data used in constructing the mathematical model (see also Fig. 2) ${ }^{\mathrm{a}}$

\begin{tabular}{|c|c|c|c|c|c|c|}
\hline \multirow[t]{2}{*}{ Step } & \multirow[t]{2}{*}{ Reaction } & \multirow[t]{2}{*}{ Kinetics } & \multirow{2}{*}{$\begin{array}{l}\text { Equilibrium constant } \\
q \text { (no unit), mass action } \\
\text { rate constant } k\left(\min ^{-1}\right)\end{array}$} & \multirow[t]{2}{*}{$K_{\mathrm{m}}(\mu \mathrm{M})$} & \multicolumn{2}{|c|}{$V_{\max }^{\prime}\left(\mathrm{nmol} \min ^{-1} 1^{-1}\right)$} \\
\hline & & & & & $\begin{array}{l}\text { MAM1 Mutant } \\
\text { (gsml-3) }\end{array}$ & Wild-type \\
\hline \multicolumn{7}{|c|}{ Chain elongation } \\
\hline $\mathrm{E}_{1}(\mathrm{MAM} 3)$ & МTOB $\rightarrow$ MTOP & \multirow{8}{*}{$\begin{array}{l}\text { Irreversible } \\
\quad \text { Michaelis-Menten }\end{array}$} & & 932 & 17.38 & 1.448 \\
\hline $\mathrm{E}_{2}(\mathrm{MAM} 3)$ & MTOP $\rightarrow$ MTOH & & & 476 & 17.94 & 1.495 \\
\hline $\mathrm{E}_{3}(\mathrm{MAM} 3)$ & МТOH $\rightarrow$ МТОНр & & & 463 & 34.43 & 2.869 \\
\hline $\mathrm{E}_{4}(\mathrm{MAM} 3)$ & MTOHp $\rightarrow$ MTOO & & & 358 & 16.8 & 1.4 \\
\hline $\mathrm{E}_{5}(\mathrm{MAM} 3)$ & MTOO $\rightarrow$ MTON & & & 253 & 4.37 & 0.364 \\
\hline $\mathrm{E}_{6}(\mathrm{MAM} 3)$ & MTON $\rightarrow$ MTOD & & & 81 & 0.37 & 0.031 \\
\hline $\mathrm{E}_{1}(\mathrm{MAM} 1)$ & MTOB $\rightarrow$ MTOP & & & 3,000 & 0 & 45 \\
\hline $\mathrm{E}_{2}(\mathrm{MAM} 1)$ & MTOP $\rightarrow$ MTOH & & & 640 & 0 & 100 \\
\hline $\mathrm{G}_{1}(\mathrm{Cyp} 79 \mathrm{~F} 1)$ & $\mathrm{MTOP} \rightarrow \mathrm{C} 3$ & \multirow{8}{*}{$v=\frac{V_{\max } * T}{\frac{K_{M}}{q}+T}$} & $q=4$ & 34 & 0.46 & \\
\hline $\mathrm{G}_{2}(\mathrm{Cyp} 79 \mathrm{~F} 1)$ & $\mathrm{MTOH} \rightarrow \mathrm{C} 4$ & & $q=1$ & 34 & 1 & \\
\hline $\mathrm{G}_{3}(\mathrm{Cyp} 79 \mathrm{~F} 1)$ & MTOHp $\rightarrow$ C5 & & $q=1$ & 37 & 0.024 & \\
\hline $\mathrm{G}_{4}(\mathrm{Cyp} 79 \mathrm{~F} 1)$ & $\mathrm{MTOO} \rightarrow \mathrm{C} 6$ & & $q=1$ & 194 & 0.04 & \\
\hline $\mathrm{G}_{5}(\mathrm{Cyp} 79 \mathrm{~F} 1)$ & $\mathrm{MTON} \rightarrow \mathrm{C} 7$ & & $q=1$ & 216 & 0.016 & \\
\hline $\mathrm{G}_{6}(\mathrm{Cyp} 79 \mathrm{~F} 1)$ & MTOD $\rightarrow$ C8 & & $q=1$ & 74 & 0.008 & \\
\hline $\mathrm{G}_{5}(\mathrm{Cyp} 79 \mathrm{~F} 2)$ & $\mathrm{MTON} \rightarrow \mathrm{C} 7$ & & $q=1$ & 374 & 0.01 & \\
\hline $\mathrm{G}_{6}(\mathrm{Cyp} 79 \mathrm{~F} 2)$ & MTOD $\rightarrow$ C8 & & $q=1$ & 26 & 0.01 & \\
\hline \multicolumn{7}{|l|}{ Degradation } \\
\hline & $\mathrm{C} 3 \rightarrow$ & Irreversible mass action & $k=7 * 10^{-7}$ & & & \\
\hline & $\mathrm{C} 4 \rightarrow$ & & $k=7 * 10^{-7}$ & & & \\
\hline & $\mathrm{C} 5 \rightarrow$ & & $k=7 * 10^{-7}$ & & & \\
\hline & $\mathrm{C} 6 \rightarrow$ & & $k=7 * 10^{-7}$ & & & \\
\hline & $\mathrm{C} 7 \rightarrow$ & & $k=7 * 10^{-7}$ & & & \\
\hline & $\mathrm{C} 8 \rightarrow$ & & $k=7 * 10^{-7}$ & & & \\
\hline
\end{tabular}

a Parameters given in roman are experimental kinetic data, while those written in italics have been optimized to fit the model. Experimental data for MAM3 was obtained in Textor et al. (2007); for MAM1 in Textor et al. (2004) and for Cyp79F1 and F2 in Chen et al. (2003). Constant concentration of the external metabolite OMTB $=1 \mu \mathrm{M}$

$E_{1}-E_{6}$ : Chain elongation of the 2-oxo acids, represented by the condensation reaction catalyzed by MAM1 and MAM3

$\mathrm{G}_{1}-\mathrm{G}_{6}$ : Core glucosinolate formation and reamination (equilibrium), represented by the aldoxime formation catalyzed by Cyp79F1 and $\mathrm{F} 2$ 
transamination reactions (which are not yet characterized) by unknown parameters, increasing computational difficulties. As transamination reactions are known to be fast and reversible (as discussed above), we can assume that the $V_{\max }$ values of the uncharacterized transamination reactions of the chainelongated 2-oxo-acids are in all probability large enough to allow the reactions to be fast and in rapid equilibrium. This reduces the number of unknown parameters significantly to one unknown parameter per reaction, the equilibrium constant $q$. Since values for the equilibrium constants of the specific transaminase reactions involved in glucosinolate biosynthesis are not available, we used a value of 4 for the transamination of the first chain-elongated 2-oxo-acid (MTOP) and a value of 1 for all further transaminations, since these optimized the model best with the actual glucosinolate distribution and are in the range of equilibrium constants of other transaminase reactions reported in the literature (Tachiki and Tochikura 1975; Cruickshank and Isherwood 1958). A similar reasoning underlies the choice of $V_{\max }^{\prime}$ for the steps from transamination to glucosinolate formation. To simplify the model, the very weak activity of MAM1 on MTOH was left out.

For the degradation reactions, simple irreversible mass action kinetics were assumed $v=k * S\left(S: \mathrm{C}_{3}\right.$, $\mathrm{C}_{4}, \ldots ; v$ : reaction rate; $k$ : rate constant). All the rate constants were assigned the same value regardless of chain length.

To model the MAM1 and MAM3 knockout mutant, we set the maximal velocities of their catalyzed reactions to zero. For the MAM1 mutant, we assumed a compensatory overexpression of MAM3. We modelled this by a 12-fold increase of the maximal activity of all MAM3 reactions, again with zero activity of MAM1.

\section{Metabolite concentrations}

The concentrations of the chain-elongated 2-oxo acids and the final glucosinolates were allowed to vary in the model and are calculated under steadystate conditions from the system equations. Their initial concentrations were set to zero. All other metabolites, in particular the initial substrate for chain elongation, MTOB, were regarded as "external metabolites" and assumed to have fixed concentrations. Note that not all of these metabolites (e.g. acetyl-CoA and the amino group donor of the transaminase) are explicitly given.

Concentrations refer to total cell volume rather than particular organelles, although glucosinolates appear to be synthesized in the chloroplasts and cytosol and stored in vacuoles. This is because the measurements were done for entire tissues rather than particular organelles. Thus, concentrations should here be interpreted as average values.

\section{Software}

Numerical calculation employed the metabolic simulation package COPASI (Hoops et al. 2006), which is freely available from http://www.copasi.org. Here the input reaction kinetics and the reaction network structure are translated automatically into ordinary differential equations.

\section{Results}

When the simulated glucosinolate distribution for the wild type Columbia computed by the model is compared to the actual glucosinolate content of this plant line measured by chemical analysis, the profiles are shown to be in good agreement with each other (Fig. 3). For the MAM1 knockout mutant, two simulations were carried out: In the first simulation, the maximal velocities of MAM1-catalyzed reactions were set to zero and the same MAM3 activity as in the wild type model was used (Fig. 3b). This resulted in the reversal of the ratio of $\mathrm{C}_{3} / \mathrm{C}_{4}$ glucosinolates as seen in the actual glucosinolate content of the MAM1 mutant. However, it led to a depletion of the $\mathrm{C}_{8}$ glucosinolates and, furthermore, to a significant reduction of the total glucosinolate content. Neither of the two latter effects is in accordance with the experimental data, which indicate a significant increase in $\mathrm{C}_{8}$ content, together with a moderate increase in total glucosinolate content. A decrease in the activity of an enzyme such as MAM1 should lead to a decrease in the steady-state concentrations of all metabolites downstream of the perturbed enzyme according to Metabolic Control Analysis (cf. Heinrich and Schuster 1996; Fell 1997), unless the pathway has unusual kinetic properties such as substrate inhibition. However, the deficiency of an enzyme in a mutant is often at least partially 

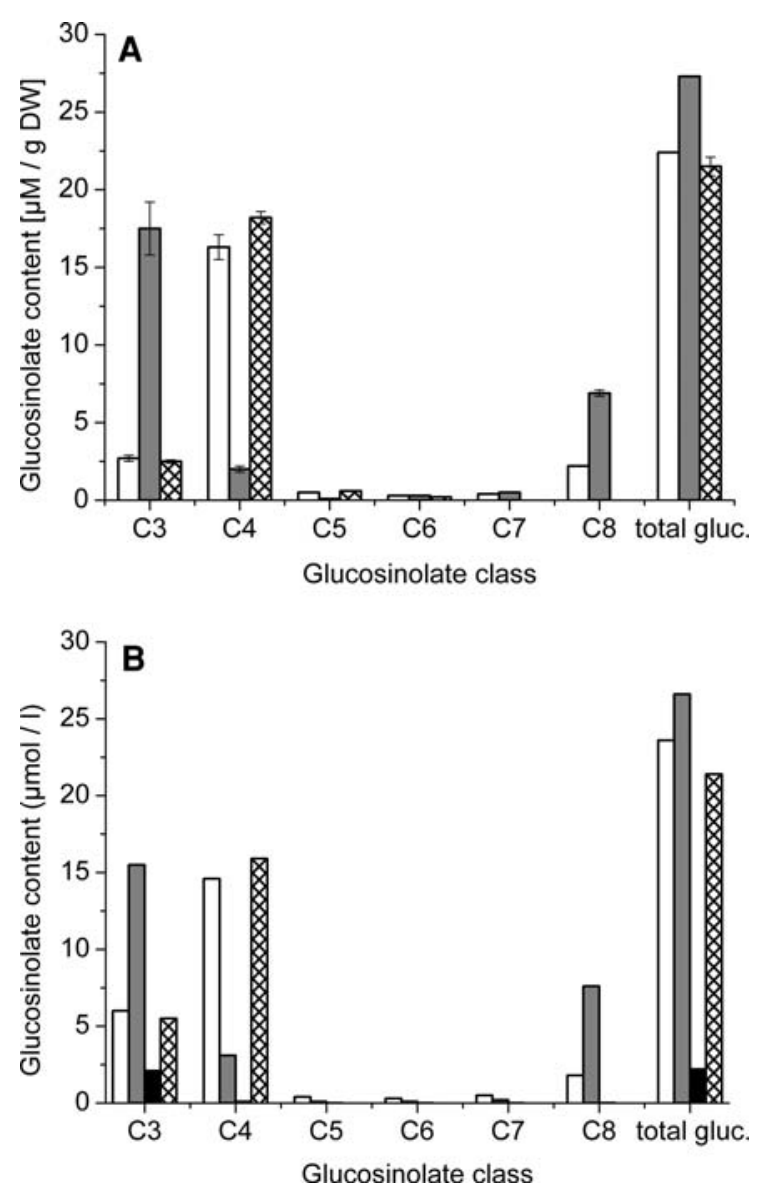

Fig. 3 Comparison of actual leaf glucosinolate profiles of plant lines to those predicted by the mathematical simulation. (a) Actual values measured from analyses of leaves of the Col0 wild-type (white bars), of the MAM1 knockout mutant gsm13 (grey bars) and the MAM3 knockout mutant gsm2-1 (grid bars). Aliphatic glucosinolates sum up to 22.4 (wild type), 27.3 (MAM1 knockout mutant) and 21.5 (MAM3 knockout mutant) $\mu \mathrm{mol} * \mathrm{gDW}^{-1} \cdot \mathrm{C}_{7}$ and $\mathrm{C}_{8}$ glucosinolates were not detected in the MAM3 knockout mutant gsm2-1. Glucosinolates were isolated, identified and quantified as described in Textor et al. (2007). Mean and standard error are based on three replicate samples. Where standard error was not depicted, it was too small to show accurately. (b) Modelled glucosinolate profile. Wild-type (white bars), MAM1 knock-out mutant with (grey bars) and without (black bars) compensation for MAM3 overexpression and MAM3 knockout mutant (grid bars)

compensated by an increase in the activity of other enzymes (e.g. Fong and Palsson 2004). This has indeed been found for MAM1 deficiency based on the elevated transcript level of MAM3 in the MAM1 mutant (Textor et al. 2007, see supplemental Fig. 3). Therefore, in our second simulation, it was assumed that the loss of MAM1 is compensated by an overexpression of $M A M 3$. With a 12-fold increase of the maximal activity of all MAM3 reactions, the predicted glucosinolate profile is now similar to the actual profile measured by direct chemical analyses. All of the three observations mentioned in the Introduction (significant increase in $\mathrm{C}_{8}$ glucosinolates, moderate increase of total glucosinolate content and reversal of the $\mathrm{C}_{3} / \mathrm{C}_{4}$ ratio) are reproduced in this simulation, and thus can be explained by a knockout of MAM1 with a compensatory overexpression of MAM3. This qualitative effect is largely independent of the exact $K_{m}$ values of MAM3, as was checked by simulations (data not shown).

Two MAM3 mutant lines, gsm2-1 (a missense mutant) and gsm2-2 (a T-DNA insertion line) show a slight increase in the proportion of $\mathrm{C}_{4}$ glucosinolates and a depletion of the longest chain glucosinolates, $\mathrm{C}_{7}$ (in gsm2-1) and $\mathrm{C}_{8}$ (in both mutants) (see Textor et al. 2007 and Fig. 3a). A simulation of a MAM3 knockout mutant, in which the maximal velocities of MAM3-catalyzed reactions were set to zero (Fig. 3b), reflected these profile changes well. The model of the MAM3 knockout mutant also showed proportions of zero for the $\mathrm{C}_{5}$ and $\mathrm{C}_{6}$ glucosinolates, while minor amounts of these compounds were observed in the actual plant analyses.

\section{Discussion}

The aliphatic glucosinolates in A. thaliana are a large group of methionine-derived secondary metabolites. Their structural diversity arises in large part from the variable length of their side chains. The corresponding metabolic network is so complex that mathematical modeling is required to try to understand it. Here, we have established a model of the biosynthesis of the aliphatic glucosinolates in A. thaliana, based on our knowledge of the structure of the pathway, the kinetic properties of its enzymes and MAM transcript levels. Given the cyclic nature of the pathway, the irregular variation in kinetic parameters for substrates of varying chain length and the involvement of two MAM enzymes, it was impossible to judge how alterations in kinetic parameters would affect the product outcome by simple inspection. Mathematical modeling provides a powerful tool to increase our understanding of metabolic networks in living cells (Heinrich and Schuster 1996; Mendes 1997). A particular challenge 
in constructing this model was dealing with enzymes having broad substrate specificities. This is a hallmark of many enzymes of secondary metabolism and contributes to the enormous diversity of products observed (e.g., Gang et al. 2002). Only a few modeling studies on such enzymes have been presented so far (Kacser and Beeby 1984; Nuño et al. 1997; Schuster and Zevedei-Oancea 2002, 2004).

Although we constructed a simplified, "skeleton" model, less than half of the necessary parameters were available or could be derived from the literature. We chose the remaining parameter values such that the model predictions for the Columbia wild-type were in good agreement with the actual glucosinolate profiles analyzed. Also the model predictions for MAM1 and MAM3 knockout mutants showed a striking agreement with the actual glucosinolate content of the respective plant lines, given the degree to which the pathway of aliphatic glucosinolate biosynthesis was simplified for modeling purposes. The simulated glucosinolate profiles show qualitatively and semi-quantitatively all the same important characteristics as the actual profiles (see Results section).

The change in glucosinolate profile of the MAMI mutant relative to wild-type is due to the remaining MAM3 activity. The MAM1 mutant exhibits a leaf glucosinolate profile involving all chain lengths $\mathrm{C}_{3}-$ $\mathrm{C}_{8}$ dominated by $\mathrm{C}_{3}$ and $\mathrm{C}_{8}$. Although a trace level of MAM1 transcript is detectable in this mutant (see Textor et al. 2007; supplemental Fig. 3), the near identity of this glucosinolate phenotype with that previously described for a MAMI missense mutant (gsm1-1, Kroymann et al. 2001) suggests that it is a virtual knock-out. The MAM3 mutant was simulated as a total knockout, lacking $\mathrm{C}_{5}-\mathrm{C}_{8}$ glucosinolates, but small amounts of these compounds are found on actual analyses. Much of the observed $\mathrm{C}_{5}$ glucosinolate content likely results from the weak activity of MAM1 for the third elongation reaction, which was omitted from the model for simplicity reasons. The small amounts of the longer aliphatic glucosinolates, $\mathrm{C}_{6}-\mathrm{C}_{8}$, for the two MAM3 mutants gsm2-1 and gsm2-2 might be due to a residual activity of MAM3.

Using the model, we were able to substantiate experimental indications that the levels of the two MAM activities are dependent on each other, as the loss of MAM1 is accompanied by a compensatory increase of MAM3. The final glucosinolate profile is thereby strongly determined by the ratio of MAM1 and
MAM3 catalytic activities on a common pool of methionine or its 2-oxo acid derivatives, suggesting that the two enzymes are located in the same subcellular compartment. Since MAM1 is predicted to be a plastidial protein and MAM3 has been proven to be so (Textor et al. 2007) this common compartment is most likely the chloroplast. Moreover, the model calculations showed that the reversal in the $\mathrm{C}_{3} / \mathrm{C}_{4}$ ratio is mainly due to the loss of MAM1 activity, while the increase in the $\mathrm{C}_{8}$ share and the total glucosinolate content in the MAM1 knock-out mutant is due to elevated levels of MAM3. As mentioned in the Results section, an increased transcript level of MAM3 in the MAM1 mutant was observed experimentally. This can be understood intuitively: the plant "attempts to" compensate for the loss of MAM1 by elevating the level of the other condensing enzyme. An open question is how this over-expression of MAM3 is facilitated at the level of the gene-regulation network. The computer simulation showed that a MAM1 mutant without increased MAM3 activity would suffer a decrease in both the proportion of $\mathrm{C}_{8}$ glucosinolates and in total glucosinolate content, in contrast to the actual plant analyses. Thus, the model turned out to be valuable in explaining the role of compensatory MAM3 expression by allowing one to separate two processes-knockout of MAM1 and compensation by $M A M 3$. These two events occur together in reality and are thus difficult to separate experimentally. Comparing the MAM1 knockout mutant without overexpression of MAM3 with the MAM3 knockout mutant shows that the MAM3 activity leads to a dominance of $\mathrm{C}_{3}$ in the glucosinolate profile, while the MAM1 activity leads to an abundance of $\mathrm{C}_{4}$.

The kinetic parameters employed in the model were measured in vitro as is typically done in metabolic modeling studies (e.g. Heinrich et al. 1977; Galazzo and Bailey 1990; Heinrich and Schuster 1996; Chassagnole et al. 2001). Accurate in vivo kinetic data for glucosinolate biosynthetic enzymes are virtually impossible to obtain in our present state of knowledge because measurement would need to be restricted to only those cells involved in glucosinolate production. In constructing our model, missing parameter values were estimated so as to optimize the agreement between the model and the system under investigation, as has also been done in many classical metabolic modeling studies. While there are often discrepancies between enzyme 
properties in vitro and in vivo (Benevolensky et al. 1994), the good agreement of the model with measured glucosinolate concentrations in wild-type and two different mutant situations suggests that in vitro kinetic data are a good approximation of in vivo enzyme behavior in this case.

Due to the gaps in our knowledge of the kinetic parameters of glucosinolate biosynthetic enzymes, we abstained from building a comprehensive kinetic model aimed at an exact quantitative simulation. Our model is nevertheless ideal for answering questions such as: Which factors lead to the three concomitant changes in glucosinolate profile observed between the wild type and MAM1 knockout mutant? Can the counter-intuitive increase in total glucosinolate content be simulated by what is known about the structure and parameters of the biochemical network? The model presented here should nevertheless be considered as a first step towards a more quantitative description of glucosinolate biosynthesis. As more experimental data become available, they will be included in future versions of the current model. It will then be important to test the reliability of the model by applying it to more variants or even different individual plants.

A further direction in which such metabolic models can be refined comes from the fact that the total enzyme concentration of a multifunctional enzyme (with broad substrate specificity) is actually distributed over the different substrates. In the case under study, the 2-oxo-acids of different chain length may compete among each other for the MAM enzyme. Therefore, the magnitude of metabolic flux, which is determined by the amount of MTOB catalyzed, may be diminished by the proportion of MAM1 and MAM3 involved in the catalysis of other chain length 2-oxo-acids. Strictly speaking, this would require deriving a more complicated enzyme kinetics because the usual Michaelis-Menten kinetics is based on the conservation relation $E+E S=$ const., while an enzyme with broad substrate specificity implies a more complicated conservation relation. This leads to an effective decrease of the enzyme amount available for each particular substrate. We can assume, however, that we have partially circumvented this problem here by the use of scaled maximal velocities $\left(V_{\max }^{\prime}\right)$.
One parameter that obviously differs between the in vivo and in vitro situations is temperature. As plants live under varying temperatures, it is hard to decide which temperature should be used for in vitro measurements. In a classical approach, this is done under conditions where enzyme activity is highest, which turned out to be $32^{\circ} \mathrm{C}$ for MAM3 in our system (Textor et al. 2007). Since a change in temperature affects most enzyme activities in a similar way, stationary metabolite levels would not be expected to change dramatically when all enzyme activities were subjected to a similar temperature change. This is mathematically expressed by the summation theorem for concentration control coefficients (cf. Heinrich and Schuster 1996; Fell 1997). A similar reasoning applies to changes in the $\mathrm{pH}$ value.

An important goal of mathematical modeling in cell biology is to derive theoretical predictions that can be verified later in experiments. However, the predictive power of theoretical biology is much more limited than that of theoretical physics so far, although some success stories exist (cf. Schuster et al. 2006a, b). Nevertheless, modeling is useful also in other ways. Mathematical models allow for a formalization and validation or falsification of hypotheses. A model allows integration of diverse experimental data and information to reveal regulatory properties of cellular networks. It helps us to better understand the role of underlying mechanisms. In the system studied here, the model showed that the overexpression of MAM3 in compensation for the knock-out mutation of MAM1 is crucial to the resulting glucosinolate profile and the increase in total glucosinolate content. Even though the simulation did not provide exact numbers for these effects, numerical calculations allowed us to argue in more concrete terms than would have been possible by purely intuitive reasoning.

Acknowledgements We thank James G. Tokuhisa, JanWillem de Kraker, Axel von Kamp and Jörn Behre for stimulating discussions. The research was supported by the German National Science Foundation (Grant GE 1126/1-3) and the Max Planck Society.

Open Access This article is distributed under the terms of the Creative Commons Attribution Noncommercial License which permits any noncommercial use, distribution, and reproduction in any medium, provided the original author(s) and source are credited. 


\section{References}

Benderoth M, Textor S, Windsor AJ, Mitchell-Olds T, Gershenzon J, Kroymann J (2006) Positive selection driving diversification in plant secondary metabolism. Proc Natl Acad Sci USA 103(24):9118-9123. doi: 10.1073/pnas.0601738103

Benderoth M, Pfalz M, Kroymann J (2008) Methylthioalkylmalate synthases: genetics, ecology \& evolution. Phytochem Rev (this issue)

Benevolensky SV, Clifton D, Fraenkel DG (1994) The effect of increased phosphoglucose isomerase on glucose metabolism in Saccharomyces cerevisiae. J Biol Chem 269:4878-4882

Chassagnole C, Raïs B, Quentin E, Fell DA, Mazat J-P (2001) An integrated study of threonine-pathway enzyme kinetics in Escherichia coli. Biochem J 356:415-423. doi:10.1042/ 0264-6021:3560415

Chen S, Glawischnig E, Jorgensen K, Naur P, Jorgensen B, Olsen CE et al (2003) CYP79F1 and CYP79F2 have distinct functions in the biosynthesis of aliphatic glucosinolates in Arabidopsis. Plant J 33:923-937. doi: 10.1046/j.1365-313X.2003.01679.x

Cruickshank DH, Isherwood FA (1958) Glutamic-alanine and glutamic-aspartic transaminases of wheat germ. Biochem J 69:189-195

Fahey JW, Zalcmann AT, Talalay P (2001) The chemical diversity and distribution of glucosinolates and isothiocyanates among plants. Phytochemistry 56:5-51. doi: 10.1016/S0031-9422(00)00316-2

Fell DA (1997) Understanding the control of metabolism. Portland Press, London

Field B, Cardon G, Traka M, Botterman J, Vancanneyt G, Mithen R (2004) Glucosinolate and amino acid biosynthesis in Arabidopsis. Plant Physiol 135:828-839. doi: 10.1104/pp.104.039347

Fong SS, Palsson BO (2004) Metabolic gene-deletion strains of Escherichia coli evolve to computationally predicted growth phenotypes. Nat Genet 36:1056-1058. doi: $10.1038 / \mathrm{ng} 1432$

Galazzo JL, Bailey JE (1990) Fermentation pathway kinetics and metabolic flux control in suspended and immobilized Saccharomyces cerevisiae. Enzyme Microb Technol 12:162-172. doi:10.1016/0141-0229(90)90033-M

Gang DR, Beuerle T, Ullmann P, Werck-Reichhart D, Pichersky E (2002) Differential production of meta hydroxylated phenylpropanoids in sweet basil peltate glandular trichomes and leaves is controlled by the activities of specific acyltransferases and hydroxylases. Plant Physiol 130:1536-1544. doi:10.1104/pp.007146

Graser G, Schneider B, Oldham NJ, Gershenzon J (2000) The methionine chain elongation pathway in the biosynthesis of glucosinolates in Eruca sativa (Brassicaceae). Arch Biochem Biophys 378:411-419. doi:10.1006/abbi.2000.1812

Grubb CD, Abel S (2006) Glucosinolate metabolism and its control. Trends Plant Sci 11(2):89-100. doi: 10.1016/j.tplants.2005.12.006

Halkier BA, Gershenzon J (2006) Biology and biochemistry of glucosinolates. Annu Rev Plant Biol 57:303-333. doi: 10.1146/annurev.arplant.57.032905.105228
Haughn GW, Davin L, Giblin M, Underhill EW (1991) Biochemical genetics of plant secondary metabolites in Arabidopsis thaliana - the glucosinolates. Plant Physiol 97:217-226

Heinrich R, Rapoport TA (1973) Linear theory of enzymatic chains; its application for the analysis of the crossover theorem and of the glycolysis of human erythrocytes. Acta Biol Med Ger 31:479-494

Heinrich R, Rapoport TA (1974) A linear steady-state treatment of enzymatic chains. General properties, control and effector strength. Eur J Biochem 42:89-95. doi: 10.1111/j.1432-1033.1974.tb03318.x

Heinrich R, Schuster S (1996) The regulation of cellular systems. Chapman and Hall, New York

Heinrich R, Rapoport SM, Rapoport TA (1977) Metabolic regulation and mathematical models. Prog Biophys Mol Biol 32:1-82. doi:10.1016/0079-6107(78)90017-2

Hoops S, Sahle S, Gauges R, Lee C, Pahle J, Simus N et al (2006) COPASI-a COmplex PAthway SImulator. Bioinformatics 22(24):3067-3074. doi:10.1093/bioinformatics/btl485

Kacser H, Beeby R (1984) Evolution of catalytic proteins or on the origin of enzyme species by means of natural selection. J Mol Evol 20:38-51. doi:10.1007/BF02101984

Kacser H, Burns JA (1973) The control of flux. Symp Soc Exp Biol 27:65-104

Kholodenko BN, Schuster S, Garcia J, Westerhoff HV, Cascante M (1998) Control analysis of metabolic systems involving quasi-equilibrium reactions. Biochim Biophys Acta 1379:337-352

Kliebenstein DJ (2008) A quantitative genetics and ecological model system: understanding the aliphatic glucosinolate biosynthetic network via QTLs. Phytochem Rev (this issue)

Klipp E, Herwig R, Kowald A, Wierling C, Lehrach H (2005) Systems biology in practice. Wiley-VCH, Weinheim

Kroymann J, Textor S, Tokuhisa JG, Falk KL, Bartram S, Gershenzon J et al (2001) A gene controlling variation in Arabidopsis glucosinolate composition is part of the methionine chain elongation pathway. Plant Physiol 127:1077-1088. doi:10.1104/pp.127.3.1077

Kroymann J, Donnerhacke S, Schnabelrauch D, Mitchell-Olds T (2003) Evolutionary dynamics of an Arabidopsis insect resistance quantitative trait locus. Proc Natl Acad Sci USA 100:14587-14592. doi:10.1073/pnas. 1734046100

Lange BM (2006) Integrative analysis of metabolic networks: from peaks to flux models? Curr Opin Plant Biol 9:220226. doi:10.1016/j.pbi.2006.03.003

Mendes P (1997) Biochemistry by numbers: simulation of biochemical pathways with Gepasi 3. Trends Biochem Sci 22:361-363. doi:10.1016/S0968-0004(97)01103-1

Morgan JA, Rhodes D (2002) Mathematical modeling of plant metabolic pathways. Metab Eng 4:80-89. doi:10.1006/ mben.2001.0211

Nuño JC, Sánchez-Valdenebro I, Pérez-Iratxeta C, MeléndezHevia E, Montero F (1997) Network organization of cell metabolism: monosaccharide interconversion. Biochem $\mathbf{J}$ 324:103-111

Schuster S, Zevedei-Oancea I (2002) Treatment of multifunctional enzymes in metabolic pathway analysis. Biophys Chem 99:63-75. doi:10.1016/S0301-4622(02)00122-9 
Schuster S, Zevedei-Oancea I (2004) Multifunctional enzymes and pathway modelling. In: Hicks MG, Kettner C (eds) Proceedings of the 1st International Beilstein workshop on experimental standard conditions of enzyme characterizations. Logos-Verlag, Berlin, pp 115-127

Schuster J, Knill T, Reichelt M, Gershenzon J, Binder S (2006a) Branched-chain aminotransferase 4 is part of the chain elongation pathway in the biosynthesis of methionine-derived glucosinolates in Arabidopsis. Plant Cell 18:2664-2679. doi:10.1105/tpc.105.039339

Schuster S, Klipp E, Marhl M (2006b) The predictive power of molecular network modelling-case studies of predictions with subsequent experimental verification. In: Eisenhaber F (ed) Discovering biomolecular mechanisms with computational biology. Landes Bioscience, Georgetown, pp 95-106

Selkov EE (1975) Stabilization of energy charge, generation of oscillations and multiple steady states in energy metabolism as a result of purely stoichiometric regulation. Eur $\mathbf{J}$ Biochem 59:151-157. doi:10.1111/j.1432-1033.1975. tb02436.x

Tachiki T, Tochikura T (1975) Further characterization of Lleucine-pyruvate transaminase from Acetobacter suboxydans. Biochim Biophys Acta 397:342-346

Textor S, Bartram S, Kroymann J, Falk KL, Hick A, Pickett JA et al (2004) Biosynthesis of methionine-derived glucosinolates in Arabidopsis thaliana: recombinant expression and characterization of methylthioalkylmalate synthase, the condensing enzyme of the chain elongation cycle. Planta 218:1026-1035. doi:10.1007/s00425-003-1184-3

Textor S, de Kraker JW, Hause B, Gershenzon J, Tokuhisa JG (2007) MAM3 catalyzes the formation of all aliphatic glucosinolate chain lengths in Arabidopsis thaliana. Plant Physiol 144:60-71. doi:10.1104/pp.106.091579

Tokuhisa J, de Kraker J-W, Textor S, Gershenzon J (2004) The biochemical and molecular origins of aliphatic glucosinolate diversity in Arabidopsis thaliana. In: Romeo JT (ed) Secondary metabolism in model systems, recent advances in phytochemistry, vol 38. Elsevier Science, Amsterdam, pp 19-38

Wittstock U, Halkier BA (2002) Glucosinolate research in the Arabidopsis era. Trends Plant Sci 7:263-270. doi:10.1016/ S1360-1385(02)02273-2

Wittstock U, Kliebenstein DJ, Lambrix V, Reichelt M, Gershenzon J (2003) Glucosinolate hydrolysis and its impact on generalist and specialist insect herbivores. In: Romeo JT (ed) Integrative phytochemistry: from ethnobotany to molecular ecology, recent advances in phytochemistry, vol 37. Elsevier Science, Amsterdam, pp 101-125 